\title{
A Case of Highly Aggressive Extraskeletal Myxoid Chondrosarcoma
}

\author{
Aaron P. Mitchell ${ }^{\mathrm{a}} \quad$ Michael Poiesz ${ }^{c} \quad$ Allen Leung ${ }^{\mathrm{b}}$ \\ aDuke University Medical Center, Internal Medicine Residency Program, Durham, \\ N.C. and ${ }^{b}$ Department of Pathology, at ${ }^{\mathrm{C}}$ NYU Langone Medical Center, New York, \\ N.Y, USA
}

\section{Key Words}

Extraskeletal myxoid chondrosarcoma - Sarcoma - Soft tissue $\cdot$ Histopathology $\cdot$ Tumor grade

\begin{abstract}
Extraskeletal myxoid chondrosarcoma (EMC) is a soft tissue malignancy characterized by specific chromosomal abnormalities involving the TEC gene. This disease has historically been considered largely indolent both histologically and clinically. Rarer subsets of EMC exist that demonstrate aggressive histopathologic features and clinical behavior, though it remains unclear whether or not aggressive histopathology is predictive of outcome. Herein we present a case of EMC with aggressive histopathologic features that underwent rapid clinical progression despite initial treatment with curative intent. This case provides the context for a discussion of the existing literature regarding treatment, prognosis, pathology, and genetic/molecular features of EMC in general and aggressive EMC specifically.
\end{abstract}

\section{Introduction}

Extraskeletal myxoid chondrosarcoma (EMC) is a rare malignancy with distinctive morphologic, histologic, and immunohistochemical features. EMC has been estimated to comprise $2.5 \%$ of soft tissue sarcomas [1]. It occurs preferentially in men at a 2:1 ratio, with $50 \%$ of all cases occurring during the 5 th or 6 th decade of life [2]. This cancer has historically been described as slow-growing and late to metastasize, with 10-year survival rates ranging from 65 to $78 \%$ [2-4].

Wide local excision is the only treatment to date that offers a potential cure for EMC [2]. Other modalities are less effective. Radiation in doses of up to $70 \mathrm{~Gy}$ has been reported to achieve substantial reduction in tumor size in individual patients [5], but this 
modality has not been associated with better outcomes in larger comparative studies [2]. No prospective trials have yet examined the effectiveness of radiation therapy for the treatment of EMC.

There is little comparative data supporting the superiority of specific chemotherapeutic regimens due to the low incidence of the disease. Generally, studies have found EMC to have a limited response to chemotherapy. Several reports have claimed responses, but these have tended to be small, such as one retrospective analysis observing 1 partial response out of only 3 patients treated with chemotherapy [6]. Larger analyses of multiple different chemotherapeutic regimens have observed no responses $[2,7]$ and a best outcome of stable disease for 6 months occurring in only $25 \%$ of patients [2].

To our knowledge, there are no studies that demonstrate a meaningfully effective systemic treatment for EMC, and no large prospective trials exist. As such, outcomes for patients with recurrent or metastatic disease that cannot be managed by local modalities remain poor, despite its slow progression. Though patients may survive for years with systemic involvement, most patients who do not achieve a surgical cure will eventually succumb to their disease [8].

EMC with high-grade histology has been reported in rare cases in the literature. It is unclear whether or not there is any relationship between high-grade histology and aggressive clinical behavior. Herein, we report on notable clinical and histopathological features of a patient diagnosed with high-grade EMC, and discuss the pathologic, prognostic, and genetic characteristics of high-grade EMC.

\section{Case Report}

A 49-year-old man without a significant past medical history presented to an outside institution in April 2009 with a painful, enlarging left flank mass. He was treated with oral analgesics for several months, before computed tomography (CT) of the abdomen in September 2009 revealed a $6.2 \times 4.5 \mathrm{~cm}$ solid mass in the subcutaneous tissue of the left flank. Pathological review of a biopsy in October 2009 characterized the lesion as high grade (FNCLCC 3/3) with numerous mitotic figures. Histology was characterized by short, anastomosing chords and strands of ovoid cells with eosinophilic cytoplasm within a myxoid matrix, areas of necrosis, and nodularity (fig. 1). Immunohistochemistry was positive for vimentin and synaptophysin, supporting the diagnosis of EMC $[4,9]$. The tumor was positive for EMA.

By November 2009 the mass had grown to $7.7 \times 7.8 \mathrm{~cm}$, but no treatment was initiated at this time. In January of 2010 the patient was referred to surgical oncology at Bellevue Hospital Center (BHC); however, he presented to the emergency department before his scheduled appointment due to uncontrollable flank pain. He came to our attention at this time.

His physical exam on arrival to BHC was notable for a large, fungating mass protruding from his posterior left flank at the costal margin. CT imaging showed that the mass had now grown to $12.4 \times 10.4$ $\times 13.2 \mathrm{~cm}$ and was invading the musculature of the abdominal wall (fig. 2 ). A retrocrural lymph node measured $2.7 \times 1.6 \mathrm{~cm}$, but it was not biopsied. All other images in January 2010, including bone scan and brain MRI, were unremarkable and not suggestive of distant metastases.

Surgical excision of the flank mass was performed in January 2010. The deep soft tissue margin was positive for malignancy. Pathology at that time showed small round blue cells (ig. 3 ). The morphology was consistent with the cellular variant for EMC, though there were none of the typical, less-cellular areas that, when present, allow definitive diagnosis of the cellular variant of EMC. Immunohistochemical staining was consistent with EMC, showing positivity for vimentin and synaptophysin (fig. 3d) [4, 9], focal positivity of neuron-specific enolase [9], and cytokeratin [3]. EMA, 
positive in the biopsy performed in 2009 , was now negative, as were S-100, desmin, GFAP, and antismooth muscle actin, also consistent with this diagnosis [10].

Several features were suggestive of high-grade histology. There were up to 10 mitotic figures per 10 high power fields, extensive areas of necrosis, and positive Ki-67 staining in over 95\% of cells (fig. 3c). Cytogenetic studies did not identify the common $\mathrm{t}(9: 22)(\mathrm{q} 22 ; \mathrm{q} 11)$ translocation, nor the other documented translocations involving 17q11, 15q21, or 3q11-12.

The patient subsequently developed a second mass located in the right flank which was removed by excisional biopsy in March 2010. Pathologic features were comparable to those of the left flank mass. He soon developed increasing pain from a rapidly growing, locally recurrent mass in his left flank. The mass was visible by imaging studies and physical examination, though no biopsy of the resection bed was performed to confirm local recurrence. Additional imaging revealed emergence of abdominal lymphadenopathy (now involving the retrocrural, para-aortic, common iliac, and external iliac nodes) and interval development of two discrete nodules in the left adrenal gland.

Further surgery was not deemed beneficial, and the patient underwent chemotherapy, first with one cycle of cisplatin and adriamycin and then with one cycle of ifosfamide, adriamycin, and vincristine, in April 2010. He continued to have rapid growth of the left flank mass as well as the nodal disease despite chemotherapy. The patient consequently deteriorated clinically and was referred for palliative radiation and transitioned to supportive care.

\section{Discussion}

\section{Relationship between Histologic Features and Clinical Behavior}

Our developing understanding of EMC is that this disease may be less indolent than previously believed, given its high rates of recurrence and propensity for lung metastasis. The single largest study of EMC found that approximately one half of patients will experience local recurrence after resection, and one half will develop metastatic disease [3]. This is in line with findings from smaller studies that a majority of patients will experience some form of recurrence after surgical treatment. Drilon et al. predicted $21 \%$ disease-free survival at 10 years [2], and Antonescu et al. observed an overall metastasis rate of $65 \%$ [11]. The development of metastasis decreases the likelihood of survival $[2,3]$, but this is variable. Median survival after detection of metastasis has been reported from 1.7 years [2] to 5.5 years [3], with individuals surviving up to 14 years after spread of EMC to the lung [8].

The vast majority of EMC cases are histologically low grade. However, several highgrade variants have been described [11]. Given the exquisite rarity of this uncommon subtype of an uncommon malignancy, high-grade EMC has been difficult to study and remains poorly characterized [5].

The tumor in this case was remarkable for its aggressive histologic appearance and clinical behavior, both of which are atypical for EMC. The initial histology on first biopsy was characteristic for EMC by morphology and surface markers. However, when surgically excised several months later, the morphology was hypercellular with no resemblance to typical EMC. This transformation suggests that dedifferentiation had occurred, a process seen not uncommonly in aggressive malignancies.

This patient had a number of factors - age $>45$ years, large primary tumor, tumor located on trunk rather than extremity - that have previously been identified as poor 
prognostic features for EMC $[3,4]$. However, it remains unclear whether histological signs of aggressive behavior, such as dedifferentiation, Ki-67 staining, mitotic rate, and necrosis provide additional prognostic information. Anecdotally, our case is remarkably similar to a previously reported case of EMC, in which excision of the primary mass was followed by growth of a recurrent, dedifferentiated tumor and rapid clinical deterioration with metastatic disease [12].

Regardless, the clinical significance of EMC tumor grade remains controversial. Several small studies of EMC found that high-grade lesions were associated with a worse prognosis. The presence of rhabdoid cells was correlated with worse outcomes in a series of 36 patients [13]. A follow-up study of 20 patients with EMC found that high-grade tumors were associated with shorter overall survival $(p=0.045)$; tumor grade showed a trend toward increased likelihood of metastasis, but this finding was not statistically significant $(\mathrm{p}=0.065)$ [11]. A study of 23 patients found that high cellularity, atypia, and the presence of focal regions of Ki-67 staining above $25 \%$ were all significant predictors of lower overall survival. Mitotic activity was correlated with lower overall survival but did not reach statistical significance [4]. A larger study of 117 patients came to different conclusions. Necrosis and Ki-67 labeling were significant predictors of metastasis, and metastasis predicted worse survival. However, neither necrosis, Ki-67 staining, high cellularity, mitotic activity, nor atypia were statistically significant independent predictors of worse prognosis [3].

The only report dedicated solely to high-grade EMC, a case series, observed survival times of 2,3.5, and 10 months from diagnosis in the 3 patients for whom full follow-up was available [5]. This study is limited by the small size of its cohort, but it supports the conclusion that the median survival for high-grade EMC is shorter than that of lowergrade disease.

\section{Genetics of EMC}

EMC genetics are typically defined by one of several chromosomal translocations involving the TEC gene. The $\mathrm{t}(9: 22)$ (q22q11) translocation and/or the resultant EWSR1/TEC fusion product is identifiable in approximately $70 \%$ of EMC cases [14]. Another fusion product, RBP56/TEC, associated with translocation $\mathrm{t}(9: 17)(\mathrm{q} 22 ; \mathrm{q} 11.2)$, is the primary mutation in an additional $15 \%$ of EMC [14]. Two additional translocations involving TEC have been described in single case reports.

Interestingly, none of these translocations were found on cytogenetic analysis in this case. While the presence of these mutations is typically confirmatory of a diagnosis of EMC, there are documented cases in which no known TEC translocation was identifiable using conventional methods. One such study of 18 EMC cases found 12 tumors with EWSR1/TEC and 3 with RBP56/TEC by RT-PCR, but 3 tumors had no identifiable fusion products [15].

These results raise the possibility that other unidentified molecular abnormalities contribute to the pathogenesis of EMC. The two most common fusion products, ESWR1/TEC and RBP56/TEC, are found in only 90\% of EMCs, leaving room for TCF12/TEC, TGF/TEC, and potentially other undescribed molecular abnormalities. This is a likely occurrence in the case presented here. 


\section{Conclusions}

EMC has been considered a largely indolent disease. Survival even after recurrence is, on average, prolonged, despite ineffective systemic therapy. However, there are reports in the literature suggesting that survival for patients who have high-grade tumors is reduced. Subsequent work has failed to demonstrate statistically significant prognostic values of individual high-grade histologic features. It is difficult to draw meaningful conclusions from these studies, given they are not adequately powered to demonstrate a small difference in prognosis between low- and high-grade disease.

This patient's tumor was both exquisitely high-grade by histologic criteria and remarkably aggressive clinically. This behavior stands in stark contrast to the indolent growth that, historically, has typified EMC. As others have, this report suggests a relationship between histologic grade and prognosis. Larger prospective case series are needed to illustrate a definitive correlation between histologic features and clinical outcomes.

Another interesting feature in this case is the absence of the typical molecular abnormalities involving the TEC gene. This contributes to a body of literature that has demonstrated that a subset of EMC patients lacks these abnormalities by initial cytogenetic analysis. Some, but not all, of these patients are found to have the characteristic or documented abnormalities following additional testing by more sensitive means. The existence of a subset of patients which demonstrates no identifiable chromosomal or molecular abnormalities by even the most sensitive existing means suggests that other, unknown molecular abnormalities may contribute to EMC. Further study is necessary to identify and characterize other possible pathogenic mechanisms, and to investigate the potential for correlation between clinical behavior and the various TEC gene translocations.

This case also reinforces early, wide local excision as the only effective treatment for EMC. In concurrence with previous studies, chemotherapy provided no observable response in this patient. Given the ineffectiveness of chemotherapy and radiation in the treatment of this disease, early and aggressive surgical resection remains the only opportunity for extended disease-free survival and a small chance of permanent disease eradication. As evidenced by prior larger patient cohorts, wide local excision in patients who present with localized disease offers a marginal but measurable chance for cure. Sadly, this patient's disease went undetected for several months, and the window during which curative resection might have been possible was missed. 

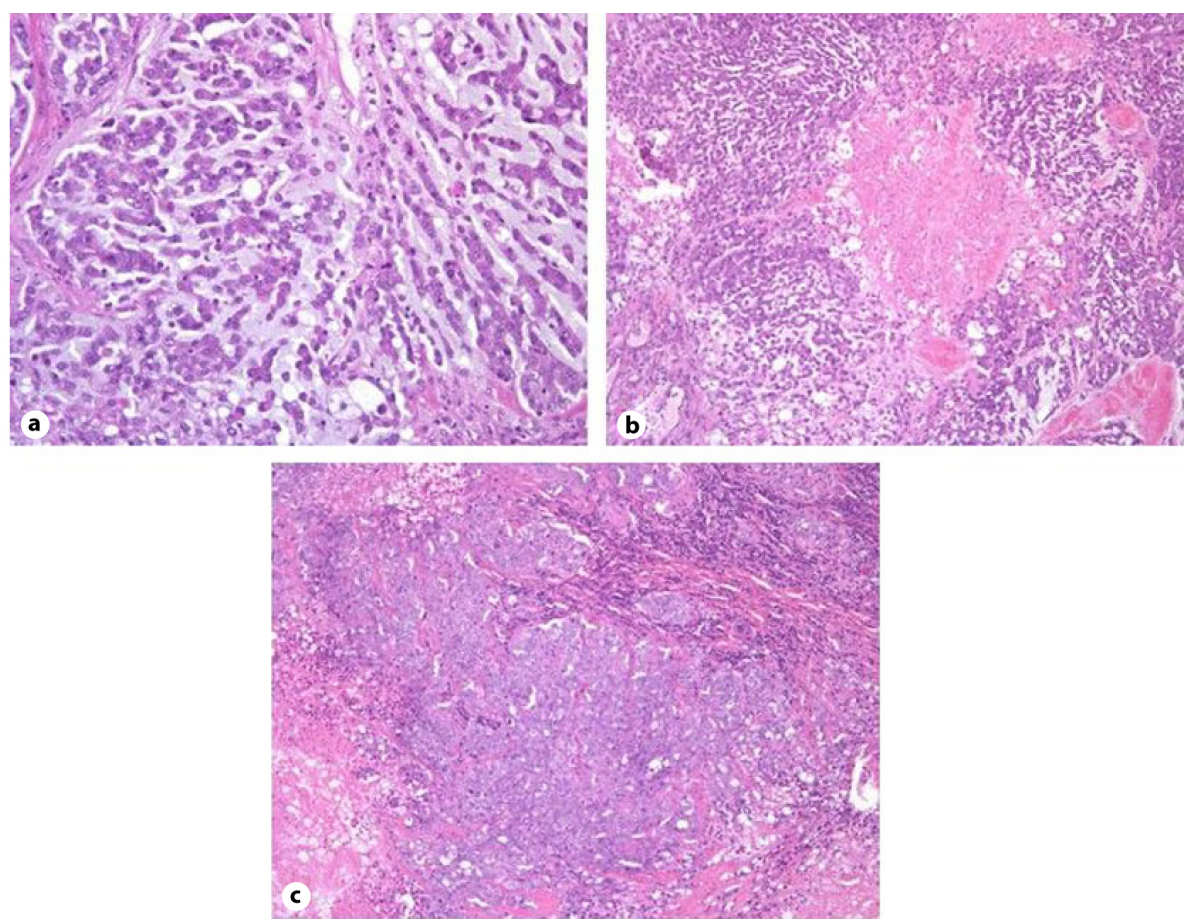

Fig. 1. Hematoxylin and eosin staining of the biopsy specimen from October 2009. Magnification: 200x (a), $100 \times(\mathbf{b}, \mathbf{c})$.
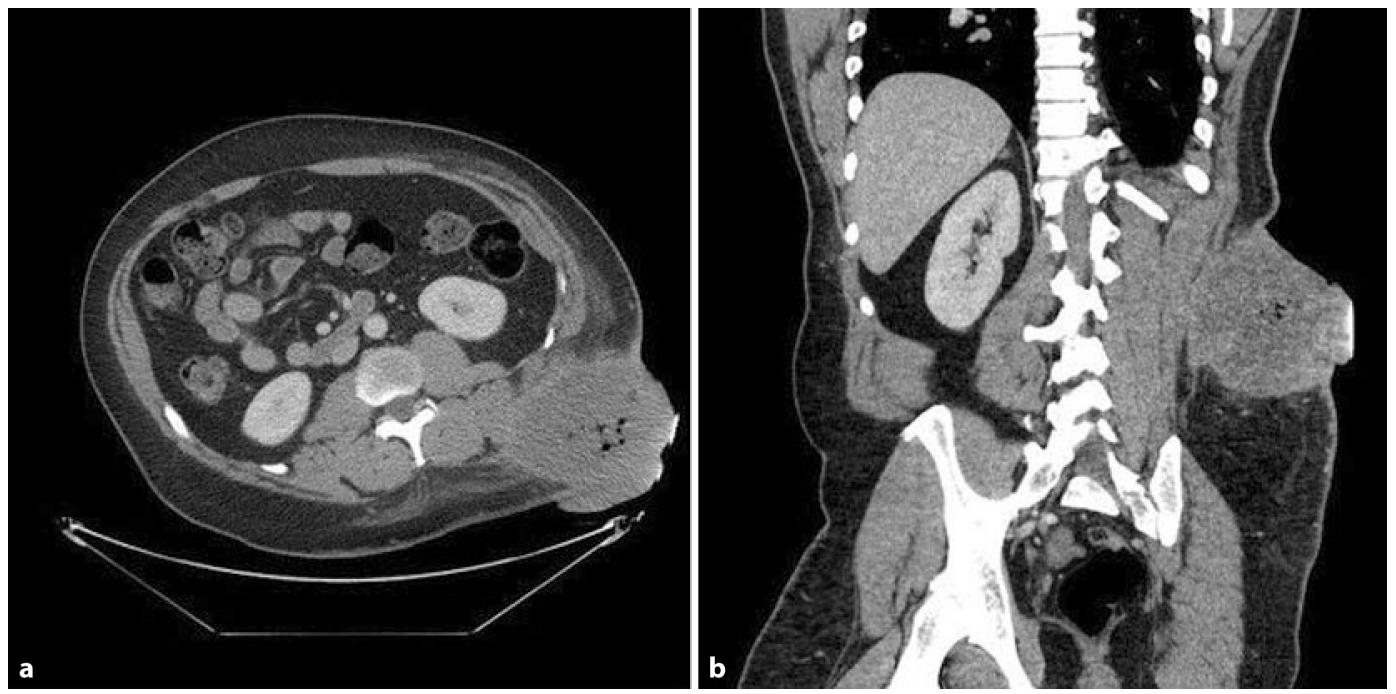

Fig. 2. a Axial and b coronal CT images from January 2010. 

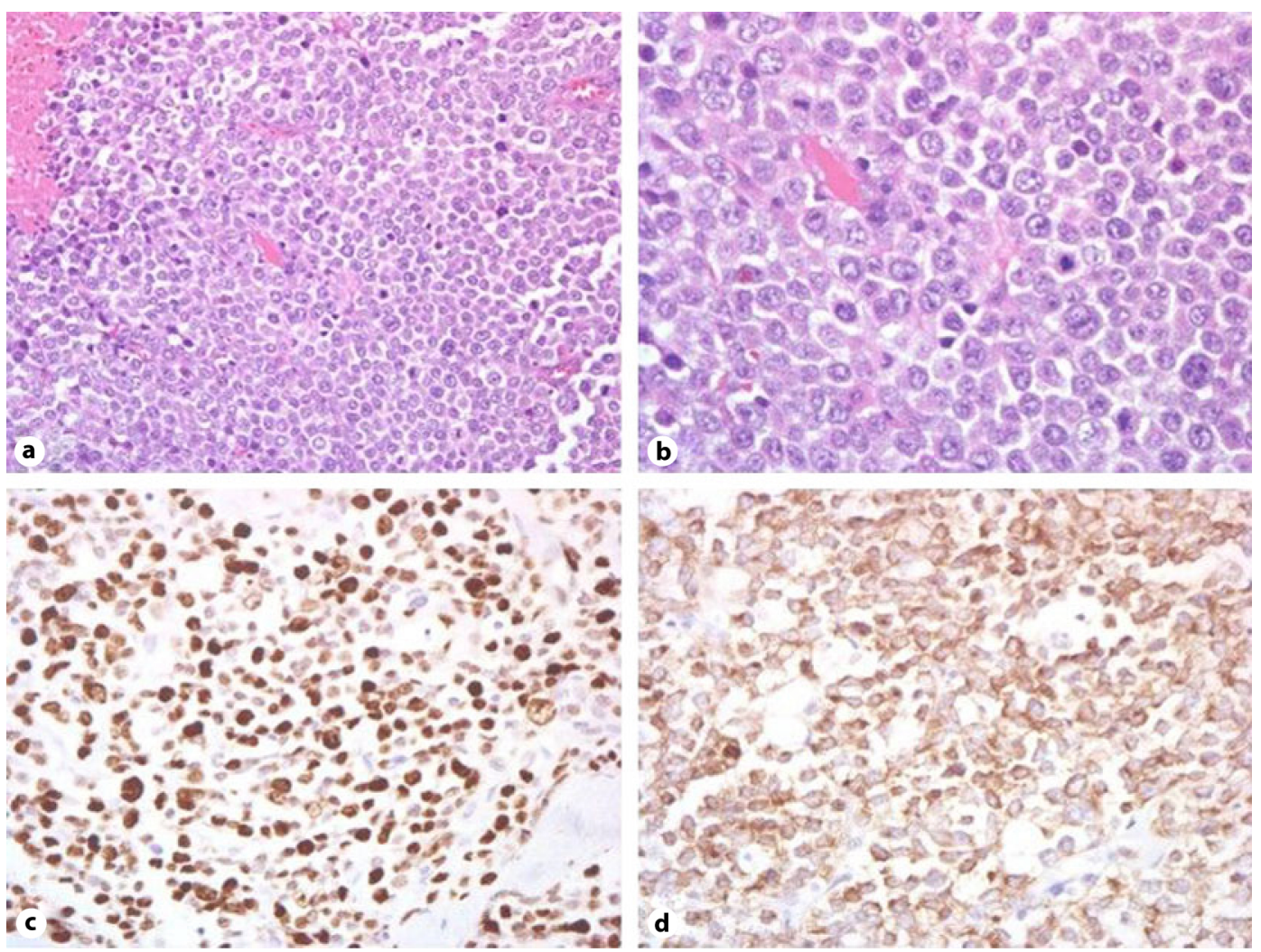

Fig. 3. a, b Hematoxylin and eosin staining of the surgical specimen from January 2010. c Ki-67 stain. d Synaptophysin stain. Magnification: $200 \times(\mathbf{a}), 400 \times(\mathbf{b}), 200 \times(\mathbf{c}, \mathbf{d})$.

\section{References}

1 Tsuneyoshi M, Enjoji M, Iwasaki H, Shinohara N: Extraskeletal myxoid chondrosarcoma - a clinicopathologic and electron microscopic study. Acta Pathol Jpn 1981;31:439-447.

-2 Drilon AD, Popat S, Bhuchar G, et al: Extraskeletal myxoid chondrosarcoma: a retrospective review from 2 referral centers emphasizing long-term outcomes with surgery and chemotherapy. Cancer 2008;113:33643371.

-3 Meis-Kindblom JM, Bergh P, Gunterberg B, Kindblom LG: Extraskeletal myxoid chondrosarcoma: a reappraisal of its morphologic spectrum and prognostic factors based on 117 cases. Am J Surg Pathol 1999;23:636-650.

4 Oliveira AM, Sebo TJ, McGrory JE, et al: Extraskeletal myxoid chondrosarcoma: a clinicopathologic, immunohistochemical, and ploidy analysis of 23 cases. Mod Pathol 2000;13:900-908.

-5 Lucas DR, Fletcher CD, Adsay NV, Zalupski MM: High-grade extraskeletal myxoid chondrosarcoma: a highgrade epithelioid malignancy. Histopathology 1999;35:201-208.

6 Kawaguchi S, Wada T, Nagoya S, et al: Extraskeletal myxoid chondrosarcoma: a multi-institutional study of 42 cases in Japan. Cancer 2003;97:1285-1292.

7 Patel SR, Burgess MA, Papadopoulos NE, Linke KA, Benjamin RS: Extraskeletal myxoid chondrosarcoma. Long-term experience with chemotherapy. Am J Clin Oncol 1995;18:161-163.

8 Saleh G, Evans HL, Ro JY, Ayala AG: Extraskeletal myxoid chondrosarcoma. A clinicopathologic study of ten patients with long-term follow-up. Cancer 1992;70:2827-2830.

-9 Goh YW, Spagnolo DV, Platten M, et al: Extraskeletal myxoid chondrosarcoma: a light microscopic, immunohistochemical, ultrastructural and immuno-ultrastructural study indicating neuroendocrine differentiation. Histopathology 2001;39:514-524. 
10 Hisaoka M, Hashimoto H: Extraskeletal myxoid chondrosarcoma: updated clinicopathological and molecular genetic characteristics. Pathol Int 2005;55:453-463.

-11 Antonescu CR, Argani P, Erlandson RA, et al: Skeletal and extraskeletal myxoid chondrosarcoma: a comparative clinicopathologic, ultrastructural, and molecular study. Cancer 1998;83:1504-1521.

12 Ramesh K, Gahukamble L, Sarma NH, al Fituri OM: Extraskeletal myxoid chondrosarcoma with dedifferentiation. Histopathology 1995;27:381-382.

13 Oshiro Y, Shiratsuchi H, Tamiya S, et al: Extraskeletal myxoid chondrosarcoma with rhabdoid features, with special reference to its aggressive behavior. Int J Surg Pathol 2000;8:145-152.

-14 Sjögren H, Meis-Kindblom JM, Orndal C, et al: Studies on the molecular pathogenesis of extraskeletal myxoid chondrosarcoma - cytogenetic, molecular genetic, and cDNA microarray analyses. Am J Pathol 2003;162:781792.

15 Okamoto S, Hisaoka M, Ishida T, et al: Extraskeletal myxoid chondrosarcoma: a clinicopathologic, immunohistochemical, and molecular analysis of 18 cases. Hum Pathol 2001;32:1116-1124. 